Provided for non-commercial research and education use. Not for reproduction, distribution or commercial use.

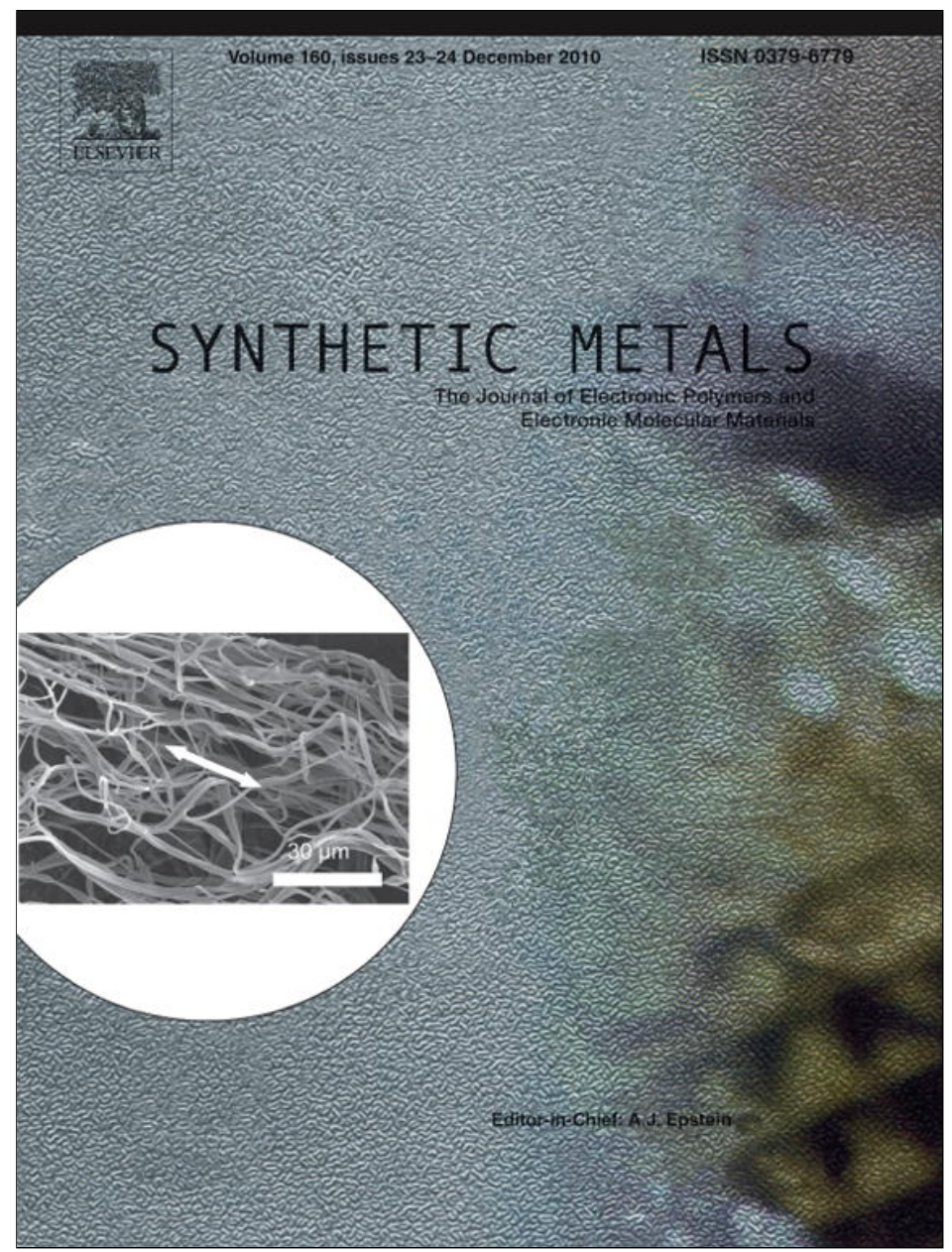

This article appeared in a journal published by Elsevier. The attached copy is furnished to the author for internal non-commercial research and education use, including for instruction at the authors institution and sharing with colleagues.

Other uses, including reproduction and distribution, or selling or licensing copies, or posting to personal, institutional or third party websites are prohibited.

In most cases authors are permitted to post their version of the article (e.g. in Word or Tex form) to their personal website or institutional repository. Authors requiring further information regarding Elsevier's archiving and manuscript policies are encouraged to visit:

http://www.elsevier.com/copyright 


\title{
Enhanced stability of poly(3-hexylthiophene) transistors with optimally cured poly(methyl methacrylate) dielectric layers
}

\author{
S.W. Lin ${ }^{\mathrm{a}, \mathrm{b}}$, Y.M. Sun ${ }^{\mathrm{a}}$, A.M. Song ${ }^{\mathrm{a}, *}$ \\ a School of Electrical and Electronic Engineering, University of Manchester, Manchester M60 1QD, United Kingdom \\ ${ }^{\mathrm{b}}$ Key Laboratory of Ministry of Education for Application Technology of Chemical Materials in Hainan Superior Resources, Hainan University, \\ Haikou 570228, People's Republic of China
}

\section{A R T I C L E I N F O}

\section{Article history:}

Received 2 May 2010

Received in revised form 3 August 2010

Accepted 18 September 2010

Available online 20 October 2010

\section{Keywords:}

Organic field-effect transistors

Air stability

Dielectrics

Polymers

\begin{abstract}
A B S T R A C T
Poly(3-hexylthiophene)-based organic field-effect transistors (OFETs) have been fabricated on poly(methyl methacrylate) (PMMA) gate dielectric layers under different process conditions, resulting in very different device stability in ambient air. The dielectric layers were prepared by spin coating and subsequently curing at various temperatures $\left(120,150\right.$, and $\left.180^{\circ} \mathrm{C}\right)$ or by ultraviolet light (UV) exposure. With respect to the variations of the on/off current ratio and the threshold voltage, dramatically enhanced stability of the OFETs with the PMMA layer cured at $150^{\circ} \mathrm{C}$ has been demonstrated when compared to those cured at different temperatures. The devices cured by UV exposure showed even more superior stability, with reliable performance in ambient air for more than 10 days. The differences in the film surface morphology were analyzed and possible mechanisms for the enhanced stability are discussed.

(C) 2010 Elsevier B.V. All rights reserved
\end{abstract}

\section{Introduction}

Soluble conjugated polymers are very attractive for use in plastic electronics because they can be deposited and processed at low temperature over a large substrate area. Among various types of polymer semiconductors, regioregural poly(3-hexylthiophene)2,5-diyl (P3HT) is the most explored due to its wide commercial availability, high carrier mobility and reasonable on/off current ratios $[1,2]$. However, it is still very challenging to fabricate and measure P3HT-based organic field-effect transistors (OFETs) in ambient conditions due to the rapid undesirable interactions of P3HT with atmospheric oxygen, moisture, ultraviolet light (UV) as well as other species in ambient air [3-5].

Efforts have been attempted to improve the device performance and reliability in air. McCulloch et al. explored strategies to chemically modify the thiophene backbone structure, which led to changes in both backbone conformation and microstructures as well as the electronic energy levels of the molecular orbitals [6]. The substitution of the central thiophene unit with naphthalene resulted in an increase in ionization potential (IP) to improve the air stability but reduce the charge carrier mobility. Recently, his group reported on new semiconducting thieno[3,2b]thiophene polymers with the enhancement in charge-carrier

\footnotetext{
* Corresponding author. Tel.: +44161306 4762.

E-mail address: A.Song@manchester.ac.uk (A.M. Song).
}

mobility and good transistor stability under static storage and operation in a low-humidity air environment due to the unusual liquid-crystalline morphological properties [7]. Also, Ong et al. reported a class of air-stable polythiophenes termed poly( $3,3^{\prime \prime \prime}-$ dialkyl-quaterthiophene)s through structural design for proper self-assembly ability and controlled $\pi$-conjugation [8].

Besides these chemical structural designs, it was reported that OFET performance depended significantly on the way the films were prepared, the kind of dielectric gate insulator used, pretreatment of the gate insulator, and the solvents used for film deposition [9-15]. Our previous work investigated in the influence of process conditions on the stability of P3HT-based OFETs [16]. An improvement in ambient-air stability, that a $120 \mathrm{~min}$ exposure to air caused a slight degradation of OFETs, was achieved via a combination of a high boiling-point solvent, a highly hydrophobic self-assembled monolayer (SAM) for dielectric surface treatment, and thermal annealing in a nitrogen gas. The experiments demonstrated that the stability of OFETs may be improved not only by optimization of the chemical structures of the organic semiconductor, but also by refining the device fabrication processes. The latter approach is quite convenient because it offers to the final users the possibility of achieving a stable OFET with the same materials only by changing the processing conditions.

In this work, we fabricated bottom-gate top-contact P3HTbased OFETs embedding a PMMA layer between the silicon dioxide dielectric and the active P3HT channel. The influence of the PMMA layers under different process conditions on the device ambientair stability has been investigated. We demonstrated that P3HT 
devices on optimally cured PMMA layers showed improved stability, with high on/off current ratio and relatively stable threshold voltage, when exposed to ambient conditions for more than 10 days. To make use of the full advantages of organic electronic devices, namely, large area coverage, mechanical flexibility, and low-cost processing, polymer gate dielectrics are the most suitable candidates in OFETs. Recently, Bao's group has demonstrated the thin poly(4-vinylphenol) (PVP) cross-linked polymer dielectric films for low-voltage organic transistors without the help of any additional inorganic insulating layers [17]. Besides PVP, PMMA is among the most promising polymeric materials due to the easy processing, high stability, and outstanding chemico-physical properties $[18,19]$. Our work about the PMMA layers cured under different process conditions here not only provides information on the effects of dielectric treatment on device properties, but also leads to important implications in realization and optimization of the performance of organic electronic devices.

\section{Experiments}

For convenience and a direct comparison with most previous studies, highly doped silicon wafers with a thermally grown $\mathrm{SiO}_{2}$ $(300 \mathrm{~nm})$ were used as substrates. Bottom-gate top-contact OFETs based on regioregular P3HT (Rieke metals, used as received) were fabricated on the PMMA buffer layer on the substrates. PMMA films were prepared from solution of PMMA (Sigma-Aldrich, molecular weight $350 \mathrm{~K}$ ) and $n$-butyl acetate, by spin-coating and subsequent curing at various temperatures or by UV exposure. For the thermal treatment, PMMA layers were cured at different temperatures $\left(120,150\right.$, and $\left.180^{\circ} \mathrm{C}\right)$ for one hour in a slightly overpressured nitrogen gas. The UV exposure was performed at the UV wavelength of $365 \mathrm{~nm}$ and the density of $18.3 \mathrm{~mW} / \mathrm{cm}^{2}$ for $1 \mathrm{~min}$. For all samples, P3HT was dissolved in 1,2,4-trichlorobenzene (TCB) at a concentration of $10 \mathrm{mg} / \mathrm{ml}$ and was spin-coated at $2000 \mathrm{rpm}$ on top of the cured PMMA layers in air. After that, the samples were annealed at $100^{\circ} \mathrm{C}$ for two hours under nitrogen. The thickness of the P3HT and PMMA layers were about $30 \mathrm{~nm}$ and $300 \mathrm{~nm}$, respectively, as measured by atomic force microscope (AFM). Finally, gold source/drain electrodes were evaporated through a shadow mask, defining $60 \mu \mathrm{m}$ long and $2 \mathrm{~mm}$ wide OFET channels. All the devices were stored in ambient air in the dark. The air stability was determined by measuring the device current-voltage $(I-V)$ characteristics over an extended time period with a semiconductor parameter analyzer (Agilent E5270B). On each kind of the PMMA layer, we fabricated nine P3HT OFET devices. All of the nine devices were measured every time. Similar OFET results could be achieved on the same kind of PMMA layer. For comparison, we selected the best results of OFETs on each kind of PMMA layer.

\section{Results and discussion}

Typical OFET transfer characteristics were measured in the saturation regime with a source-drain voltage of $V_{S D}=-80 \mathrm{~V}$. Fig. 1 shows the $I-V$ results of P3HT OFETs on the PMMA dielectric layers under different process conditions. The time dependence of the device performance presents different trends. For clarity, the transfer characteristics measured after $4 \mathrm{~h}$ were not included in Fig. 1 due to no difference observed from those of the as-prepared devices. When compared to our previous results with air stability of about $120 \mathrm{~min}$ on the $n$-octadecyltrichlorosilane (OTS) treated $\mathrm{SiO}_{2}$ dielectric layer in Ref. [16], vast improvement in device stability was observed on OFETs prepared on the PMMA dielectric layers, with only a slight degradation of OFETs when subjected to ambient air for up to a few days. A summary of the performance of the as-prepared P3HT OFET is given in Table 1, including field-effect mobility, threshold voltage, on/off current ratio, and subthreshold slope. For each gate interface structure, we also measured the total gate dielectric capacitance using an Agilent E4980 LCR meter and the values (at $10^{6} \mathrm{~Hz}$ ) are shown in Table 1 as well.
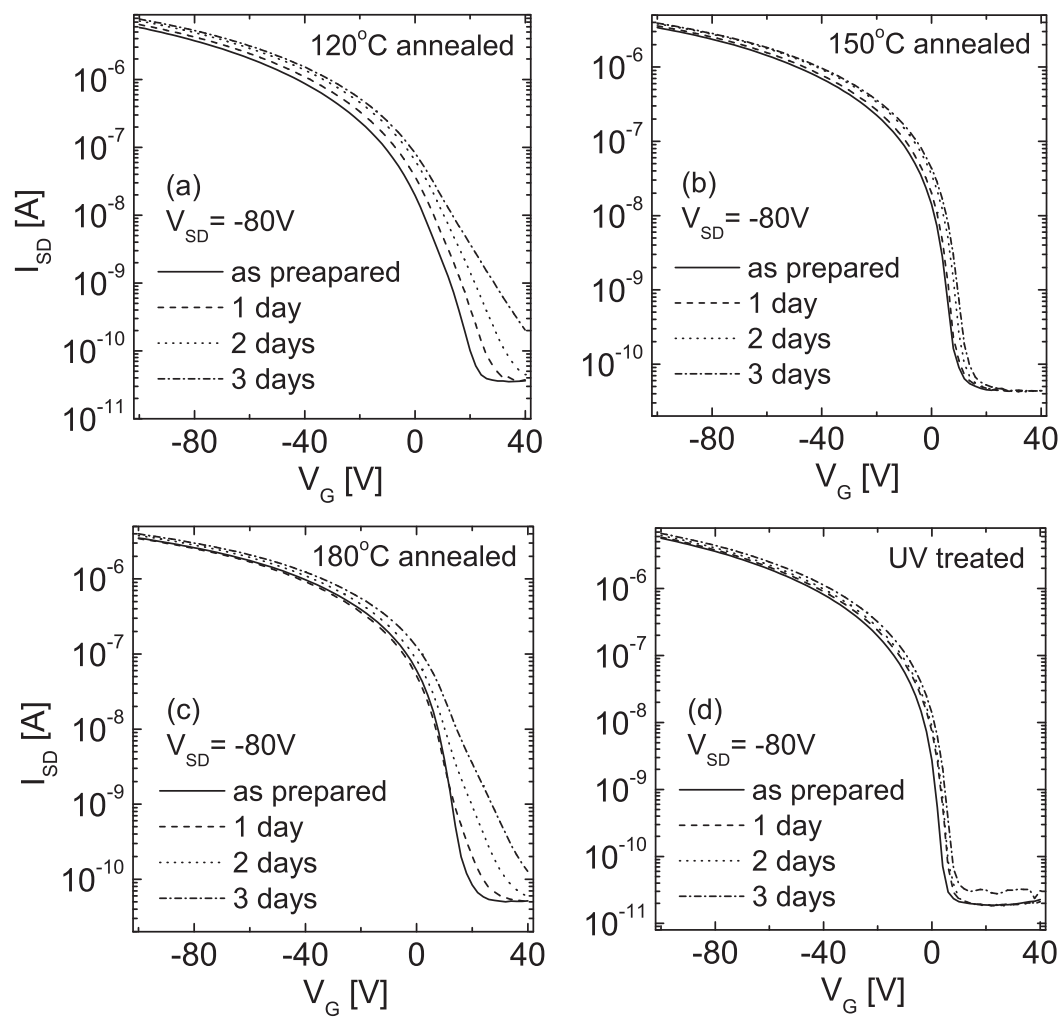

Fig. 1. Ambient-air stability of P3HT-based OFETs with the PMMA gate dielectric layers cured at (a) $120^{\circ} \mathrm{C}$, (b) $150^{\circ} \mathrm{C}$, (c) $180^{\circ} \mathrm{C}$, and by (d) UV treatment. 
Table 1

Field-effect mobility, threshold voltage, on/off ratio, and subthreshold slope of as-prepared P3HT OFETs deposited on PMMA dielectric layers with different process conditions.

\begin{tabular}{|c|c|c|c|c|c|}
\hline PMMA process condition & Capacitance $\left(\mathrm{nF} / \mathrm{cm}^{2}\right)$ & $\mu\left(\mathrm{cm}^{2} \mathrm{~V}^{-1} \mathrm{~s}^{-1}\right)$ & $V_{\mathrm{T}}(\mathrm{V})$ & $I_{\mathrm{on}} / I_{\mathrm{off}}{ }^{\mathrm{a}}$ & $S$ (V/decade) \\
\hline $120^{\circ} \mathrm{C}$ annealed & 41.8 & 0.0087 & -3.1 & $1.6 \times 10^{5}$ & 6.8 \\
\hline $150^{\circ} \mathrm{C}$ annealed & 43.6 & 0.0045 & 6.0 & $1.0 \times 10^{5}$ & 3.2 \\
\hline $180^{\circ} \mathrm{C}$ annealed & 44.7 & 0.0045 & 12.9 & $6.8 \times 10^{4}$ & 4.2 \\
\hline UV treated & 48.2 & 0.0074 & -3.7 & $2.5 \times 10^{5}$ & 2.3 \\
\hline
\end{tabular}

a The on/off ratio for all samples was calculated at the gate voltages of $-100 \mathrm{~V}$ and $40 \mathrm{~V}$.

As shown in Fig. 1(a), the OFETs with the PMMA layer prepared at $120^{\circ} \mathrm{C}$ had a lower threshold voltage $V_{\mathrm{T}}=-3.1 \mathrm{~V}$, but a larger (inverse) subthreshold slope $S=6.8 \mathrm{~V} /$ decade, than the OFETs prepared under the other conditions. $V_{\mathrm{T}}$ is usually determined by the doping level in the active channel while $S$ is associated with the carrier trap density at the interface between the active layer and the dielectric layer. The mobility in the saturation regime was determined as $0.0087 \mathrm{~cm}^{2} / \mathrm{Vs}$ [5]. The mobility value was higher than or comparable to the values reported for such kind of P3HT in ambient air [20]. From the transfer curves measured after different storage time, the device was doped in days, which still demonstrated better air stability than those on OTS-treated substrates as in our previous report [16].

The stability was found to be dramatically improved in OFETS with the PMMA dielectric layer prepared at a higher temperature like $150^{\circ} \mathrm{C}$ as shown in Fig. 1(b). Little change in the transfer characteristics was observed even after 3 days. The mobility, $\mu=0.0045 \mathrm{~cm}^{2} / \mathrm{Vs}$, was a little lower than that of the OFETs with the PMMA layer cured at $120^{\circ} \mathrm{C}$ but typical for devices produced by this kind of $\mathrm{P} 3 \mathrm{HT}$ in ambient air [20]. It is interesting to observe that the threshold voltage, $V_{\mathrm{T}}=6 \mathrm{~V}$, increased while the subthreshold slope, $S=3.2 \mathrm{~V} /$ decade, decreased with the PMMA curing temperature. The increased $V_{\mathrm{T}}$ suggests a relatively high doping level in the as-prepared devices as they were transferred from the vacuum chamber to the measurement setup, which normally took about $10 \mathrm{~min}$. The improved $S$ indicates that there were much fewer carrier traps at the interface between the PMMA and P3HT active layers [21]. The results thus might suggest that charge trap density at the interface plays an important role in the OFET air stability.

For the devices on the PMMA layer cured at an even higher temperature of $180^{\circ} \mathrm{C}$, as shown in Fig. $1(\mathrm{c})$, the threshold voltage dramatically increased to $12.9 \mathrm{~V}$ while the subthreshold slope slightly increased to $4.2 \mathrm{~V} /$ decade. Much more doping might be expected in the P3HT layer, which can be evidenced by AFM measurements later. The off current apparently increased when the devices were stored for more than 1 day, indicating an inferior stability in comparison to the results of OFETs with the PMMA layer prepared at $150^{\circ} \mathrm{C}$. Given the above results, we might think that $150^{\circ} \mathrm{C}$ is the optimal PMMA-curing temperature to improve


Fig. 2. Atomic force micrographs of P3HT films on the PMMA dielectric layers processed under different conditions: (a) $120{ }^{\circ} \mathrm{C}$ annealed, (b) $150{ }^{\circ} \mathrm{C}$ annealed, (c) $180^{\circ} \mathrm{C}$ annealed, and (d) UV treated. All the micrographs are in $2 \times 2 \mu \mathrm{m}^{2}$. 
the stability of P3HT OFETs with the reasonable mobility of $0.0045 \mathrm{~cm}^{2} / \mathrm{Vs}$ and high on/off current ratio of $10^{5}$.

We further examine the P3HT-based OFETs on the PMMA dielectric layer which was treated by UV exposure instead of thermal treatment. As shown in Fig. 1(d), the air stability was comparable to the devices on the PMMA layer annealed at $150^{\circ} \mathrm{C}$. Most importantly, the mobility could reach $0.0074 \mathrm{~cm}^{2} / \mathrm{Vs}$, and on/off current ratio was well above $10^{5}$. The device switched on around $0 \mathrm{~V}\left(V_{\mathrm{T}}=-3.7 \mathrm{~V}\right)$ and $S$ was nicely reduced to $2.3 \mathrm{~V} /$ decade. A relatively high and stable OFET performance has been demonstrated for P3HT on PMMA films cured by UV exposure, implying the polymeric dielectric layer could be optimized to realize full organic electronic devices.

To gain an insight into the stability differences, the P3HT films were imaged using an atomic force microscope in the tapping mode. PMMA layers under different process conditions have also been imaged before coating the P3HT layer. Very smooth surface with the area fluctuation RMS less than $0.6 \mathrm{~nm}$ was observed for all the PMMA layers cured under different conditions. Such indicates that the difference at the interface between the P3HT and PMMA layers might come from the interaction of the two layers rather than from the morphology and performance of the PMMA layer only. As shown in Fig. 2(a)-(c), the area fluctuation RMS and the maximum peak-to-valley fluctuation $Z_{\max }$ increased with increasing temperatures. The P3HT film on the PMMA layer cured at $120^{\circ} \mathrm{C}$ in Fig. 2(a) appeared relatively more amorphous and uniform, which suggests good connectivity among the P3HT polymers and hence a high fieldeffect mobility [12]. The device stability, however, was inferior. This is understandable because the glass transition temperature $\left(T_{\mathrm{g}}\right)$ of PMMA is $122^{\circ} \mathrm{C}$ and the curing temperature of $120^{\circ} \mathrm{C}$ is not sufficient to densely pack the PMMA film. During the P3HT film spin-coating process, the PMMA layer was dissolved by TCB solvent, which could be observed as the swelling on the P3HT surface by an optical microscope (not shown here). This thus led to a less crystalline or an amorphous film, as shown in Fig. 2(a), and a large carrier trap density at the interface between the active channel and the insufficiently cured PMMA layer, which is associated with a relatively high subthreshold voltage $S=6.8 \mathrm{~V} /$ decade. And the large trap density at the interface causes the quick deterioration of the OFETs when subjected to ambient air.

The thermal treatment of the polymeric layer is generally required to conduct at a temperature higher than $T_{\mathrm{g}}$ by $20-30^{\circ} \mathrm{C}$ for reducing the free volume formed inside the layer during spin coating and facilitating the polymer chains to move freely and to be effectively cross-linked [22]. Hence, a compact PMMA layer could be achieved as raised the curing temperatures to $150^{\circ} \mathrm{C}$, and no swelling and dissolution by TCB over the time scale of the experiment was observed. Fig. 2(b) shows apparent boundaries of the formed P3HT crystalline domains, corresponding to the increased roughness RMS of $1.95 \mathrm{~nm}$ and larger $Z_{\max }$ of $13.1 \mathrm{~nm}$. This caused a lower mobility than that of the OFETs with the PMMA layer cured at $120^{\circ} \mathrm{C}$ due to the increasing charge carrier scattering [23]. Because of the tightly packed P3HT molecules, it was very difficult for oxygen and water molecules to further penetrate through the domains, thereby resulting in a more sustained stability as shown in Fig. 1(b).

When the PMMA buffer layer was cured at an even higher temperature of $180^{\circ} \mathrm{C}$, rather rough surface $\left(Z_{\max }\right.$ is $\left.14.8 \mathrm{~nm}\right)$ was observed in Fig. 2(c). A few deep pinholes were formed in the P3HT layer and might facilitate oxygen and water molecules penetrating the film and doping the P3HT at the semiconductor-insulator interface, where the hole transport took place. This resulted in a larger $V_{\mathrm{T}}$ of $12.9 \mathrm{~V}$ and a low stability as compared to the devices with the PMMA layer cured at $150^{\circ} \mathrm{C}$. The observation demonstrates the importance of interfacial interactions on both the microstructure of the active layers and the performance of P3HT OFETs, which is in agreement with the recent report by Sethuraman et al. [12].
The results above show that both the top active layer quality and the carrier trap density at the interface play a key role in the device degradation mechanism $[1,20,21]$. To achieve high device mobility and stable performance, two important aspects should be concerned. Firstly, the connectivity of the P3HT molecules and the uniformity of the active layer not only play an important role in the field-effect mobility, but also affect the vulnerability of the active layer to the penetration of the oxygen and moisture in air. Secondly, in the bottom-gate top-contact OFET structure, the nanocrystalline islands in the P3HT films nucleate from the dielectric interface. The reliable device performance requires a compact and less defective polymeric buffer layer, that is, a low charge-trap density at the interface.

The P3HT film on the PMMA layer cured by UV exposure satisfies these two considerations and has an excellent combination of high carrier mobility and enhanced air stability. As shown in Fig. 2(d), its RMS was $0.32 \mathrm{~nm}$ and $Z_{\max }$ was $2.57 \mathrm{~nm}$, which were far smaller than those of the surfaces in the other three micrographs. The better film uniformity not only led to a mobility increase, but also dramatically enhanced the air stability. The low carrier-trap density at the interface, indicated by the small $S$ value as shown in Table 1 , was another reason for the improved stability. It has to be mentioned that because of the lack of the sufficiently thermal treatment, the dissolution by TCB also occurred in the UV-cured PMMA layer. Similar swelling in the P3HT layer could be observed by optical microscope as that with the PMMA layer cured at $120^{\circ} \mathrm{C}$. However, the low subthreshold voltage of $2.3 \mathrm{~V} /$ decade indicates that the carrier trap density at the interface between the active channel and the UV-cured PMMA layer is much lower than that at the inter-
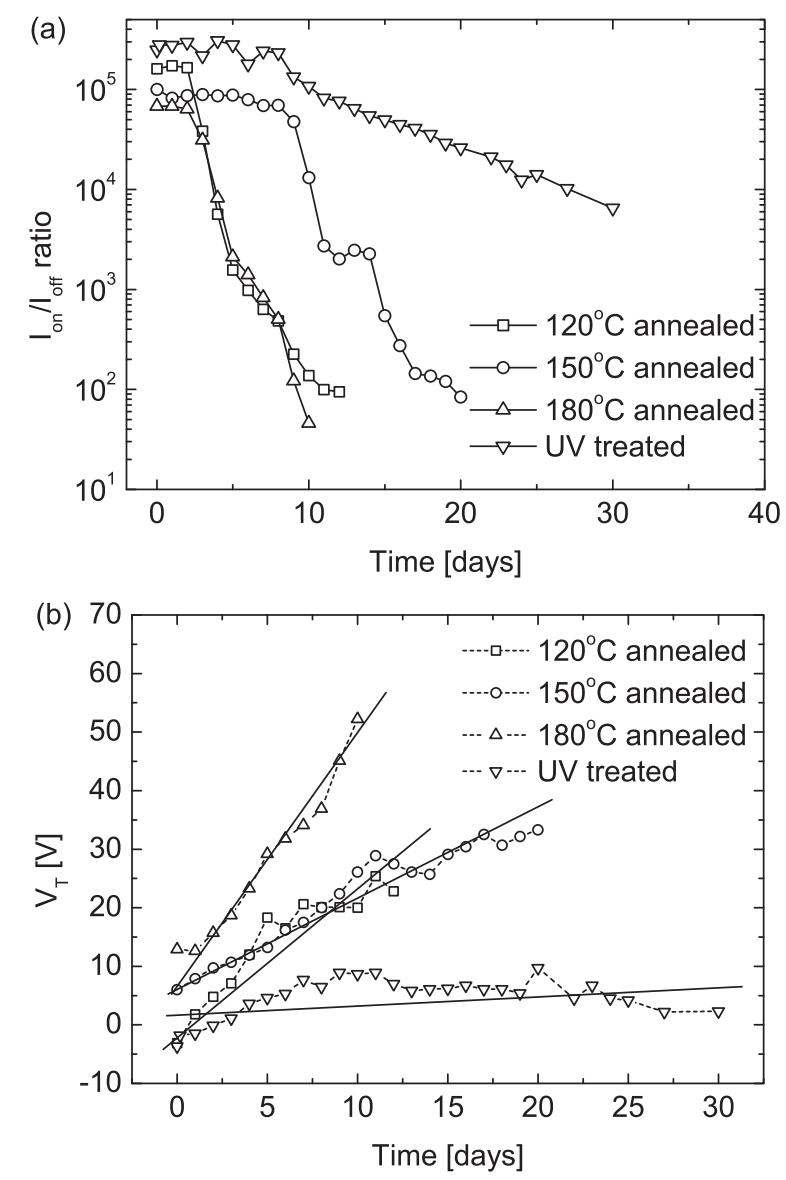

Fig. 3. The plot of (a) $I_{\mathrm{on}} / I_{\mathrm{off}}$ current ratio and (b) threshold voltage as a function of exposure time in an ambient condition. The fitted dash lines in (b) show that $V_{\mathrm{T}}$ proportionally increases as time passes. 
face between the P3HT layer and the PMMA layer cured at $120^{\circ} \mathrm{C}$. The reason is not clear but may be related to the effects of the UV exposure on the PMMA surface. New method like high-resolution transmission electron microscopy could provide more information on the interaction of the PMMA and P3HT layers, which might give useful insight into the physical understanding of the effect on the device performance.

Fig. 3 shows the $I_{\text {on }} / I_{\text {off }}$ current ratio and threshold voltage as a function of exposure time to an ambient condition in an even longer time scale. All the P3HT OFET devices were stored in ambient air in the dark, and measured over an extended time period. When the $I_{\text {on }} / I_{\text {off }}$ current ratio decreases over two orders of magnitude, we stopped the measurements. OFETs on the UV-cured PMMA layer had rather stable $I_{\text {on }} / I_{\text {off }}$ current ratio over $10^{5}$ for more than 10 days while those on the PMMA layers cured at 120 and $180^{\circ} \mathrm{C}$ deteriorated only after 2 days as shown in Fig. 3(a). The devices on PMMA cured at $150^{\circ} \mathrm{C}$ showed the moderate stability with respect to the variation of the $I_{\text {on }} / I_{\text {off }}$ current ratio. The time dependence of the threshold voltage, shown in Fig. 3(b), presents the similar trends. The slope of the fitted line in Fig. $3(\mathrm{~b})$ indicates the $V_{\mathrm{T}}$ variation rate with time. In contrast to the relatively fast increase of $V_{\mathrm{T}}$ for OFETs with the thermal annealed PMMA layers, the devices with the UV-treated PMMA layer showed a stable $V_{\mathrm{T}}$ with little change for about 10 days.

\section{Conclusions}

In summary, enhanced stability has been demonstrated in the P3HT-based OFETs with optimally cured PMMA dielectric layers. In the bottom-gate top-contact OFET structure, both the quality in the top active layer and the charge trap density at the interface influence the device air stability. Comparing the experimental results of the OFETs with the PMMA layers cured by thermal treatment and UV exposure, the P3HT film on the PMMA layer cured by the latter method possessed good film uniformity in the active layer and less carrier trap density at the interface. This thus accounted for the high and stable OFET performance with high on/off current ratio over $10^{5}$ and relatively stable $V_{\mathrm{T}}$ even after the storage time of 10 days. To achieve the full organic electronic devices, further investigation should be carried out, such as examining the OFET performance with only one layer of polymeric dielectric layer, clarifying the influence of UV treatment on the interface states, etc.

\section{Acknowledgements}

The authors would like to thank our colleagues Y. Luo and $\mathrm{H}$. Wen for useful discussion and help. This work was supported by a Royal Society Brian Mercer Feasibility Award, EPSRC, Nano ePrint Ltd. and Hainan Natural Science Foundation (807001).

\section{References}

[1] Z. Bao, A. Dodabalapur, A.J. Lovinger, Appl. Phys. Lett. 69 (1996) 4108

[2] H. Sirringhaus, P.J. Brown, R.H. Friend, M.M. Nielsen, K. Bechgaard, B.M.W Langeveld-Voss, A.J.H. Spiering, R.A.J. Janssen, E.W. Meijer, P. Herwig, D.M. de Leeuw, Nature (London) 401 (1999) 685.

[3] M.S.A. Abdou, F.P. Orfino, Y. Son, S. Holdcroft, J. Am. Chem. Soc. 119 (1997) 4518.

[4] S. Hoshino, M. Yoshida, S. Uemura, T. Kodzasa, N. Takada, T. Kamata, K. Yase, J. Appl. Phys. 95 (2004) 5088.

[5] H. Sirringhaus, Adv. Mater. 17 (2005) 2411.

[6] I. Mcculloch, C. Bailey, M. Giles, M. Heeney, I. Love, M. Shkunov, D. Sparrowe, S. Tierney, Chem. Mater. 17 (2005) 1381.

[7] I. McCulloch, M. Heeney, C. Bailey, K. Genevicius, I. MacDonald, M. Shkunov, D. Sparrowe, S. Tierney, R. Wagner, W.M. Zhang, M.L. Chabinyc, R.J. Kline, M.D. McGehee, M.F. Toney, Nat. Mater. 5 (2006) 328.

[8] B.S. Ong, Y. Wu, P. Liu, S. Gardner, J. Am. Chem. Soc. 126 (2004) 3378

[9] J. Veres, S.D. Ogier, S.W. Leeming, D.C. Cupertino, S.M. Khaffaf, Adv. Funct. Mater 13 (2003) 199.

[10] J. Chang, B. Sun, D.W. Beiby, M.M. Nielsen, T.I. Sölling, M. Giles, I. Mcculloch, H. Sirringhaus, Chem. Mater. 16 (2004) 4772

[11] I.N. Hulea, S. Fratini, H. Xie, C.L. Mulder, N.N. Iossad, G. Rastelli, S. Ciuchi, A.F. Morpurgo, Nat. Mater. 5 (2006) 982.

[12] K. Sethuraman, S. Ochiai, K. Kojima, T. Mizutani, Appl. Phys. Lett. 92 (2008) 183302

[13] J. Veres, S. Ogier, G. Lloyd, Chem. Mater. 16 (2004) 4543.

[14] C. Di, G. Yu, Y. Liu, Y. Guo, X. Sun, J. Zheng, Y. Wen, Y. Wang, W. Wu, D. Zhu, Phys. Chem. Chem. Phys. 11 (2009) 7268.

[15] R.P. Ortiz, A. Facchetti, T.J. Marks, Chem. Rev. 110 (2010) 205.

[16] L.A. Majewski, J.W. Kingsley, C. Balocco, A.M. Song, Appl. Phys. Lett. 88 (2006) 222108.

[17] M.E. Roberts, N. Queraltó, S.C.B. Mannsfeld, B.N. Reinecke, W. Knoll, Z. Bao, Chem. Mater. 21 (2009) 2292.

[18] P.M. Remiro, C. Marieta, C.C. Riccardi, I. Mondragon, Polymer 42 (2001) 9909.

[19] S. Gross, D. Camozzo, V.D. Noto, L. Armelao, E. Tondello, Eur. Polym. J. 43 (2007) 673.

[20] D.D. Schroepfer, P.P. Ruden, Y. Xia, C.D. Frisbie, S.E. Shaheen, Appl. Phys. Lett 92 (2008) 013305

[21] M. McDowell, I.G. Hill, J.E. McDermott, S.L. Bernasek, J. Schwartz, Appl. Phys. Lett. 88 (2006) 073505

[22] D.K. Hwang, J.H. Park, J. Lee, J. Choi, J.H. Kim, E. Kim, S. Im, J. Electrochem. Soc 153 (1) (2006) G23.

[23] S.J. Kang, M. Noh, D.S. Park, H.J. Kim, C.N. Whang, C.H. Chang, J. Appl. Phys. 95 (2004) 2293. 